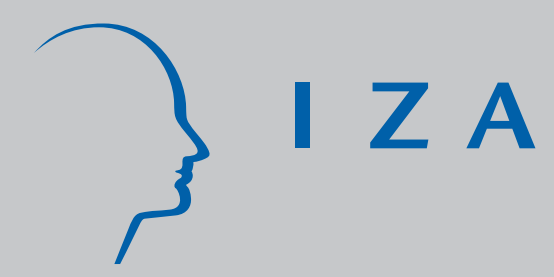

IZADP No. 2944

Interdependency in Performance

Kelii H. Haraguchi

Glen R. Waddell

J uly 2007 


\title{
Interdependency in Performance
}

\author{
Kelii H. Haraguchi \\ University of Oregon \\ Glen R. Waddell \\ University of Oregon \\ and IZA
}

\section{Discussion Paper No. 2944 \\ July 2007}

\author{
IZA \\ P.O. Box 7240 \\ 53072 Bonn \\ Germany \\ Phone: +49-228-3894-0 \\ Fax: +49-228-3894-180 \\ E-mail: iza@iza.org
}

\begin{abstract}
Any opinions expressed here are those of the author(s) and not those of the institute. Research disseminated by IZA may include views on policy, but the institute itself takes no institutional policy positions.
\end{abstract}

The Institute for the Study of Labor (IZA) in Bonn is a local and virtual international research center and a place of communication between science, politics and business. IZA is an independent nonprofit company supported by Deutsche Post World Net. The center is associated with the University of Bonn and offers a stimulating research environment through its research networks, research support, and visitors and doctoral programs. IZA engages in (i) original and internationally competitive research in all fields of labor economics, (ii) development of policy concepts, and (iii) dissemination of research results and concepts to the interested public.

IZA Discussion Papers often represent preliminary work and are circulated to encourage discussion. Citation of such a paper should account for its provisional character. A revised version may be available directly from the author. 
IZA Discussion Paper No. 2944

July 2007

\section{ABSTRACT}

\section{Interdependency in Performance}

We empirically model performance in the final round of a multiple-round tournament as a spatially autoregressive process, allowing us to sign and quantify the endogenous interactions between competitors. Doing so speaks to significant regularities in the data that suggest that a player's own performance generally tends to improve with the improving performance of competitors. However, we also find significant asymmetries in the interdependency of player performance that suggest that social interactions, even those found in a fairly straightforward game, can be rather complex. For example, while the positive complementarity in performance is particularly strong between tied players, own performance suffers in response to improving performance of lagging, lower-ability competitors.

JEL Classification: $\quad \mathrm{J33}, \mathrm{J4}, \mathrm{C} 21$

Keywords: $\quad$ peer effects, tournament, spatial, best response

Corresponding author:

Glen R. Waddell

Department of Economics

University of Oregon

Eugene, OR 97403-1285

USA

E-mail: waddell@uoregon.edu

\footnotetext{
* We thank Joe Stone for helpful comments and Ronald Davies and Wesley Wilson for beneficial discussions on earlier drafts. We accept full responsibility for any remaining errors or omissions.
} 
While much has been learned from the peer effect and social interaction literatures, relatively little attention has been paid to the potential interdependencies in player performance in competitive settings. We contribute to understanding such relationships as we document patterns of performance that are strongly suggestive of real interactions among competitors. The complexity of the relationships evident in the data suggest that fruitful research might consider more fully the potential asymmetries in how players best respond to each other's performance in competitive environments.

The existence of peer effects implies that one’s peers can influence one's own outcome. It is this potential interdependency that we address through our empirical analysis. In broad terms, Manski (2000) discusses how social interactions might affect preferences, constraints, and/or expectations of another person with resulting effects on their behavior. For example, classroom peer environments can offer temptations or distractions that make it harder for students to complete their schoolwork. Likewise, repeated or long interactions in such environments may afford the opportunity for learning from peers or for each other's sense of what is important to be influenced, indirectly affecting achievement or performance.

In our analysis we exploit data available from the Professional Golf Association’s (PGA) 2004 Tour. With that said, our hope is that this will not be viewed as just another golf paper, per se, for in several ways these data should not be discounted and offer a valuable proving ground. For example, compared to many other environments, the observed peer environments are relatively simple in nature and are short-lived, both lessening the opportunity for confounding effects to materialize. Further, the prize structures for each tournament are well defined, the cardinal ranking of players is observable, and reasonable measures of tournament and player 
characteristics that influence cardinal rankings are readily available. With such a data source, we are also able to rule out certain factors as contributing to peer effects. ${ }^{1}$

First, we suspect that key information is common across players (e.g., the rules are clear and well understood, relative standing is updated continuously) and that any observed regularity is not due to information spillovers. Second, preferences are likely to be relatively simple and unchanged over the sequence of interactions (e.g., performance is not measured differently across players, the concept of what constitutes superior performance is common). Third, given the technology of the competitive task being undertaken, we posit that there are no opportunities for learning from competitor-peers. In the end, to the extent we document interactions in player performance we will be inclined toward assuming that it is not explained by heterogeneity in preferences (in the cross section of players or over time for individual players) or "on the job” learning, leaving an appeal to differences in noncognitive attributes among potential explanations.

In the following section, we provide some discussion of the existing literature and the relevant context for our analysis. We then provide some quick set up of an empirical model that easily provides a comparison to existing results in the literature. Namely, as the literature is somewhat divided on the coefficient associated with monetary returns, Section 2 provides this result for the data assembled, but acknowledges the difficulty in interpreting such estimates in this and previous literature and quickly moves to the focus of our analysis - estimating the slope of player best-response functions.

In Section 3, we provide a brief theoretical context for examining potential interdependencies in player performance within a commonly understood tournament setting,

\footnotetext{
${ }^{1}$ There are a variety of ways to parameterize a model of peer effects, a useful taxonomy of which is provided in Hoxby and Weingarth (2006) where they discuss several competing models. The thrust of the discussion in the literature, however, is often with respect to the policy ramifications of various models (e.g., linear-in-means, shining light, bad apple, boutique, single crossing).
} 
noting that the explicit modeling of a representative dynamic game is beyond the scope of this paper. In Section 4 we discuss an empirical strategy that exploits the proximity of competitors of certain type. However, it is in our last step toward the full specification that we make our most significant contribution as we set up a spatial econometric empirical model that exploits the contemporaneous performance of competitors of different type. Answering whether players perform differently when facing different types of competitor and whether players perform differently when competitors perform differently, our identification of significant response differentials suggests that it may be overly restrictive in some settings to have in mind a model of tournament play that precludes heterogeneous best responses across tournament participants. ${ }^{2}$ We share some concluding remarks in Section 5.

\section{Context and placement in existing literature}

Important social interactions have been documented empirically in a variety of arenas. For example, Glaeser, Sacerdote, and Scheinkman (1996) has been widely cited as arguing that social interactions explain a significant portion of the variation in community crime rates. Steinberg, Brown, and Dornbusch (1996) goes as far as arguing that peer groups are more important in predicting high school performance than are parental influences. As is often the case in the literature, however, the peer effect is identified off of variation in aggregates, which neither requires that all individual-level interactions work in a common fashion nor speaks to the degree to which one-on-one interactions might matter. Our data permit the analysis of interactions at much-less-aggregate levels. While analyses of roommate peer effects often exploit individual level interactions (e.g., Sacerdote,

\footnotetext{
2 See also, McMillen, Singell and Waddell (2007), Viladecans-Marsal (2004), Brueckner and Saavedra (2001) and Brueckner (1998) for evidence of spatially dependent competition in other settings.
} 
2001; Zimmerman, 2001) they must also accept a certain complexity to the peer relationship being observed that leaves the working of the peer effect unrevealed. ${ }^{3}$

While somewhat tangential to our focus, Case and Katz (1991) document an increased probability of social ills in one neighborhood where social ills in adjacent neighborhoods are more prevalent, which is of interest here insofar as the literature is otherwise lacking in papers that analyze contemporaneous outcomes. ${ }^{4}$ For example, roommate peer effects are typically identified with covariation in a roommate's SAT score prior to college admission and own GPA, without exploiting the contemporaneous relationship between roommate GPAs. In their context, Case and Katz implicitly absorb the contemporaneous in a way that we also intend.

Turning to empirical tournament literature, we note a growing body of work that focuses on testing theoretical predictions. Adopting an experimental strategy, Bull, Schotter and Weigelt (1987) find that in competition between players of equal ability, effort levels approximate those predicted by theory. In asymmetric tournaments, however, they observe that the effort levels of disadvantaged players are higher than predicted. Tong and Leung (2002) extend this analysis to two-player, multiple-round tournaments in which each agent is informed of his opponent's output after each round. They find that average effort levels are higher in multiple-round tournaments than in single-round tournaments. Notable for our analysis due to the source of data, Ehrenberg and Bognanno (1990a, 1990b) also test the propositions of tournament theory, reporting that average player performance in professional golf tournaments improves with higher prize spreads. In a replication exercise, however, Orszag (1994) reports no significant effect of prize spread on performance.

\footnotetext{
${ }^{3}$ For example, there is an implicit first stage that often goes unmodeled that might speak to the choice of interaction and the intensity of interaction.

${ }^{4}$ In a related paper, Brock and Durlauf (2001) model individual choice in the presence of social interactions, documenting equilibrium properties and ultimately deriving an estimable likelihood function in a discrete choice framework.
} 
Before we introduce our new results we will first weigh in on these previously documented results, ultimately re-affirming that player performance improves with higher marginal returns to performance. In so doing, we caution the reader of the interpretive difficulties associated with these and previous results. In short, many hold that the set of available instruments is insufficient to cope with the endogeneity of return-type measures that tend to be the focus in the related literature. We acknowledge this criticism (here, and again below) and further emphasize that our focus is not on revisiting the relationship between effort and marginal rewards.

Having made this obligatory comparison we then make our departure into new territory by modeling player performance as spatially autoregressive. That is, we will determine the extent to which variation in one's performance is determined by the contemporaneous performance of one's close competitors. Using data from Arabian horse races, Lynch (2005) finds that race times fall with smaller variance in race times. This relates much more closely to our analysis than the Ehrenberg and Bognanno analyses, for example, as it represents the first formal evidence that contemporaneous cardinal differences in the performance of competitors determine the tournament outcomes. While this evidence does not point to asymmetries in players' best responses, it is consistent with our results insofar as we find that one's performance generally tends to correlate positively with that of one's close competitors.

Through our analysis we learn of the following empirical regularities. First, we find that the performance of one's competitors is significant in determining one's own performance, with performance tending to improve on average as that of close competitors improves. (Below we will subsequently define what we mean by "close” competitors.) Second, we find strong asymmetries in the interdependency of player performance, both across leading or lagging competitors and across ability classifications. For example, with respect to competitors of similar 
ability we find performance to be more sensitive to the play of tied competitors than to leading competitors, and not sensitive to those trailing in the tournament. The only inverse performance relationship we observe in the data is with respect to competitors of lower ability, and, in particular, lagging lower-ability competitors. That is, our analysis suggests that as the performance of lower-ability competitors improves, one's own performance declines. This is in stark contrast to the performance-enhancing effect of lower-ability but leading competitors.

\section{Data and Baseline Specification}

As discussed above, our empirical strategy will ultimately focus on estimating the slope of player best-response functions. We first present a standard OLS specification without acknowledging the potential for strategic interactions between players, and then focus on specifying a spatially autoregressive model of performance that explicitly accounts for strategic interactions. Along the way, we discuss the data and empirical specifications, the endogeneity of player performance and associated econometric concerns.

\subsection{Data}

We have assembled an original data set containing performance information for the 2004 tour of the Professional Golf Association (PGA). This collection is similar to others used in previous empirical analyses. It represents the entire 2004 season with the exception of three "major" tournaments and 14 tournaments in which the payoff structure differs from the norm. ${ }^{5}$

Key variables of interest include all player scores for each round across 35 tournaments, measures of course difficulty, weather condition at the time of the event, and the prize structure for each tournament. Controlling for player-specific skill will be important in our subsequent tests, so we

\footnotetext{
${ }^{5}$ In particular, we exclude tournaments from the analysis if a tournament consists of more than four rounds, if a cut is not made after the second round of play, if rewards are determined by methods other than the rank-ordering of total strokes or if professionals and amateurs are mixed within the field of players.
} 
also assemble a comparable data for the entire 2003 PGA Tour, which will allow all variation in outcomes from the previous season to control for relative player ability. For example, in predicting final-round performance in tournament $t$, we will use data from the 2003 PGA Tour to control for $i$ 's scoring average, putting average, proportion of greens made in regulation, and fraction of cuts made. ${ }^{6}$ We obtain all player-specific variables from the PGA, including coursespecific variables such as par and yardage. ${ }^{7}$ The Golf Course Superintendents Association of America provided another course variable, rating, which measures the difficulty of the course and is the average score on the course of all rounds played by scratch golfers. In addition, we control for any time trends in player scores by including the week in which the tournament was played, and it's square to capture non-linearity in trends, and weather information on the day of play that includes temperature and the maximum sustained wind speed. ${ }^{8}$

\subsection{Baseline specification}

Testing the standard tournament-theory hypothesis regarding player responses to marginal effort rewards is relatively straight forward. It pays, however, to first consider the three sources of variation that can be exploited for such purposes. First, within each tournament, the monetary return to effort will differ across players' ordinal rankings. That is, two differently ranked players heading into the final round of a given tournament will face different returns to effort depending on both ordinal rank and on their particular cardinal separation from their closest competitors. Second, the marginal return to effort will vary across tournaments for a given player - due to the player finding himself at a different rank in one tournament than in another. Third, given variation

\footnotetext{
${ }^{6}$ While previous studies have also included such measures, they have done so using contemporaneous outcomes (i.e., from the same season). By adopting lagged measures of ability from the 2003 Tour we avoid the simultaneity bias introduced by including contemporaneous measures.

${ }^{7}$ The official webpage of the PGA Tour can be found at pgatour.com.

${ }^{8}$ Data accessed in December 2005 from weatheralmanac.com.
} 
in the total purse awarded across tournaments, being in the same ordinal position going in to the final round will leave the player with a higher monetary return to a change in rank when the total purse is larger, which introduces variation in rewards holding ordinal rank constant. ${ }^{9}$

Our baseline specification controls for all observable characteristics available in our dataset. In subsequent specifications, we will later absorb all other time-invariant player heterogeneity into the error structure. As such, we initially specify the following model of finalround (i.e., fourth-round) scores, $S_{i t}$, for player $i$ in tournament $t$ :

$$
S_{i t}=\alpha+\beta_{0} R E T U R N_{i t}+\beta_{1} P_{i}+\beta_{2} T_{t}+\varepsilon_{i t},
$$

where we compute each player's monetary $R E T U R N_{i t}$ to improving by a single stroke assuming that all other player-scores in $t$ are unchanged. That is, for each player-tournament observation we specifically calculate the change in monetary reward that would be associated with a one-stroke improvement, accounting for the possibilities that such a change may or may not yield a change in player $i$ 's ordinal rank in tournament $t$, may or may not break a tie in ordinal rank between $i$ and other players, and may or may not create a tie between $i$ and other players. ${ }^{10}$ Across all tournaments, we intend for $P_{i}$ to capture observable player characteristics (i.e., exemption status and tour affiliation as of 2004) and for $T_{t}$ to capture observable tournament characteristics (i.e., course rating, par, yardage). In [1], $\varepsilon_{i t}$ is a traditional error term. (Unlike previous literature, our contribution does not relate to the estimated coefficient on $R E T U R N$, and we are content to

\footnotetext{
${ }^{9}$ The PGA Tour has adopted a standard formula for the allocation of monetary prizes to ranks. For example, in all tournaments in our sample, first place is always awarded 18 percent of the total purse, second place is awarded 10.8 percent, 6.8 percent, 4.8 percent, etc.

${ }^{10}$ Others have exploited variation across tournaments in the total purse, noting that prizes paid to a player of a given ordinal rank are based on a fixed percent of the tournament-specific purse. For a given rank, a larger total purse therefore generally implies a larger marginal return to effort. However, there is some evidence suggesting that purse is not independent of course difficulty. Further, we contend that once the margin has been included in the model (calculated for an individual player at his particular rank, taking into account the contemporaneous distribution of players around him) there is little else of interest in the variation in purse across tournaments. While all specifications are robust to the inclusion of purse size, we limit our attention to our preferred measure of the marginal return.
} 
assume that the potential shortcomings of this identification strategy are limited to interpreting only this coefficient and not the estimated interactions of player performance. See below for additional discussion.)

\subsection{Baseline results}

Results shown in Table 1 suggest that player scores are decreasing (i.e., are better) with greater marginal returns to effort. This confirms with our data a fundamental hypothesis of tournament models that has received mixed empirical support in the past - re-affirming the reported result of Ehrenberg and Bognanno (1990b). In addition, we document, trivially perhaps, that scores increase with course difficulty, as measured by par and yardage. Higher maximum sustained wind speeds also lead to higher scores, while temperatures below 60 degrees tend to improve scores on average.

While the inclusion of each player’s 2004 exemption status and tour affiliation should absorb at least some of the variation in player ability, it could be easily argued that there is important heterogeneity in player ability beyond that picked up by these controls. Thus, we absorb all time-invariant heterogeneity that is specific to a player across $t$ with the inclusion of player fixed effects, with the results reported in Column (2), where the estimated RETURN coefficient is smaller but remains significant. Player fixed effects should also absorb any timeinvariant heterogeneity in non-tournament returns that may influence performance (advertising revenue, for example).

Before we continue with our focus on the potential for interdependencies in final-round performance, we pause to consider the formulation of our baseline specification in one important dimension. Namely, as has been recognized in earlier literature (e.g., Ehrenberg and Bognanno, 1990), within a given tournament, a player's rank at the beginning of the final round, and therefore 
his RETURN to performance, is determined by his own past performance. Of course, in predicting final-round performance, one's performance in the first three rounds is predetermined and could therefore be treated as exogenous. ${ }^{11}$ However, if performance in the first three rounds correlates with an ability that we have not held constant in our estimation strategy, this would ultimately be absorbed in the error term of [1]. Given that we absorb all time-invariant unobserved heterogeneity specific to players into the error structure directly, we are not much troubled by this possibility, at least in general. However, were ability to be time varying for a given player, as would be the case if one can just have a "bad weekend," for example, player fixed effects would not adequately account for this. Thus, we acknowledge that even with player fixed effects, RETURN may well be endogenous, as is true of its use in previous literature.

Employing an instrumental variables technique, we exploit as instrumental variables all available tournament-day information (i.e., maximum temperature, maximum wind speed, precipitation level, and average humidity on each of the first three days of the tournament), as well as player-specific information (i.e., scoring average, fraction of cuts made, scoring average precut, and third round scoring average over the 2003 PGA Tour). Further, we include the tournament purse, and stroke totals from each of first three rounds as instruments for RETURN. ${ }^{12}$ As evident in columns (3) and (4) of Table 1, the results are robust to this treatment. However, one is wise to acknowledge that even with this IV method, one should be cautious in interpreting estimated coefficients on RETURN. It is this difficulty in the several studies that have focused on

\footnotetext{
${ }^{11}$ Note that our specifications do not include first- through third-round scores as dependent variables. As such, considering these predetermined scores as exogenous is legitimate, even in specifications that control for unobserved heterogeneity in player performance.

${ }^{12}$ In predicting final-round performance, one's performance in each of the first three rounds is exogenous to the extent that it is predetermined. Conditional on the set of included determinants and all unobserved time-invariant heterogeneity across players, there seems to be little reason to believe that there would be significant correlation remaining between the error term and RETURN. In unreported specifications, we follow Ehrenberg and Bognanno and instrument for player-scores in each of the first three rounds and include them as regressors in the second stage. Results are qualitatively similar and available upon request.
} 
measuring the RETURN coefficient that can have one also tempted to abandon our analysis. Yet, our contribution does not hang on this estimated coefficient.

\section{Theoretical context}

We are expressly interested in empirically identifying the variation in performance as it relates to that of one's competition. In particular, we will exploit observed player heterogeneity (in ability, for example) to question whether competitors interact differently with players of different type. While modeling the intricacies of a dynamic tournament with heterogeneous players is beyond the scope of this paper, the theoretical ambiguity motivating our empirical analysis is illustrated by considering a modification of Lazear and Rosen (1981) even though it is admittedly somewhat of an abstraction here. ${ }^{13}$

Lazear and Rosen (1981) document the comparative-static results of a Principal-Agent model in which realized compensation is a function of the Agent's ordinal rank - the winner being determined randomly in equilibrium from among a set of homogeneous tournament participants. Within such a framework, an agent exerts costly effort to increase the probability of winning, and thus the probability of receiving a larger prize. ${ }^{14}$ The assumption of homogeneous agents results in symmetry on multiple dimensions. First, agents choose common effort levels in equilibrium where effort is higher when prizes for winning are larger. Second, agent best-response functions are symmetric, implying that players respond similarly to out of equilibrium events.

\footnotetext{
${ }^{13}$ For discussion of dynamic tournaments see Aoyagi (2003) and Radner (1985).

${ }^{14}$ Exploiting the agent's incentive to choose higher effort levels for correspondingly higher expected payoffs, the Principal can increase the agent's effort in two ways: by increasing the ex ante probability an individual player wins (e.g., adopting a tournament with fewer players), or by increasing the spread between individual tournament prizes (which increases the marginal reward to winning the tournament while holding constant the probability of winning). When the agents' effort levels are unobservable, when relative outputs are less costly to measure than are absolute outputs, or when directly monitoring productivity is costly to the Principal, a compensation scheme based on rankorder may be less costly to implement. Further, as such costs increase, effort levels converge to those in an observable-effort scenario.
} 
In such a framework, the agent's objective function can be written generally as an expected utility,

$$
E\left[U_{i}\left(e_{i}, e_{j} ; W, X_{i}\right)\right] \equiv F,
$$

where $e_{i}$ and $e_{j}$ denote the efforts of agents $i$ and $j$, respectively, and $W$ represents the reward for winning the two-player tournament. We capture the potential for player heterogeneity in $X_{i}$, a vector of unspecified characteristics that may influence the agent's expected payoff. In general, the player's expected net return to effort is dependent on the cost of effort at the margin, the spread between tournament prizes (e.g., the monetary return associated with winning in a twoplayer game, or with an increase in ordinal rank more generally), and player-specific characteristics. However, it is also dependent on the mapping of efforts into the probabilities of various outcomes - own effort and that of other tournament participants. It is this last facet of the player's problem on which we focus.

Assuming that agents compete strategically according to Nash behavior, agent $i$ chooses effort, $e_{i}$, to maximize [2], taking as given the effort choices of competitors - just agent $j$ in the two-player tournament. The first-order condition is then an implicit function of $e_{i}$ and $e_{j}$ such as:

$$
\frac{\partial E\left[U_{i}\right]}{\partial e_{i}} \equiv F_{e_{i}}\left(e_{i}, e_{j} ; W, X_{i}\right)=0
$$

Solving for $e_{i}$, agent $i$ 's effort depends on $e_{j}, W$, and $X_{i}$, and can be expressed as $e_{i}^{*}=R\left(e_{j} ; W, X_{i}\right)$, a function that yields player $i$ 's best response to the effort choice of player $i$ 's competitor, $j$.

With the objective being to explore the interdependency of player performance in tournaments, our empirical strategy will focus on estimating the slope of this best-response function. However, before doing so, we note the theoretical ambiguity at the root of such an 
undertaking. Applying the implicit function theorem to [3], the slope of the best-response

function is given by

$$
\frac{\partial e_{i}}{\partial e_{j}}=-\frac{F_{e_{i}, e_{j}}}{F_{e_{i}, e_{i}}} .
$$

Assuming an interior solution, $F_{e_{i}, e_{i}}$ must be negative where the second-order condition is satisfied.

However, $F_{e_{i}, e_{j}}$ is ambiguous in sign and depends on the mapping of $e_{i}$ and $e_{j}$ into the probability

that $i$ wins the tournament. If an increase in $e_{j}$ raises the marginal utility of $e_{i}$ then $F_{e_{i}, e_{j}}$ is

positive, and $i$ 's reaction function is an upward-sloping function of $e_{j}$. Conversely, if an increase

in $e_{j}$ decreases the marginal utility of $e_{i}$ then the reaction function is downward sloping. If the

probability of winning, $P$, is logistic in effort levels, such that

$$
P_{i}\left(e_{i}, e_{j}\right)=\frac{e_{i}}{e_{i}+e_{j}}
$$

then $P_{e_{i}, e_{j}}=\frac{e_{i}-e_{j}}{\left(e_{i}+e_{j}\right)^{3}}$, which implies a non-monotonicity in the marginal probability of $i$ winning

the tournament as a function of $j$ 's effort. ${ }^{15}$ Given that the only value to one's effort is through

influencing this probability, the non-monotonicity of $P_{e_{i}}$ in $e_{j}$ implies that $F_{e_{i}}$ is itself non-

monotonic in $e_{j}$. It is this type of ambiguity that motivates, in part, our empirical analysis.

\footnotetext{
${ }^{15}$ Without explicitly modeling the uncertainty as in Lazear and Rosen (1981), where one’s performance is additively separable in effort and a noise parameter, this serves to illustrate the relevant ambiguity. It is more common, though, in the related strand of contest literature. Tullock (1980) first developed the model of rent seeking in which two homogeneous agents undertake costly investments in order to win a contest and its associated prize. The emphasis in Tullock-type models is more often on the efficiency of contests, rent dissipation, and social welfare, with variations on this basic theoretical model to include imperfect discrimination, larger numbers of agents, asymmetry in agents' technology, contests of multiple rounds, and multiple prizes.
} 


\section{Does One's Competition Matter?}

\subsection{Relevant competition}

To consider the ambiguity inherent to the best-responses requires that we define the set of relevant competitors for each player. As our dependent variable is final-round performance, we base this designation on the rank order of players as of the end of three rounds (i.e., the rank order at the commencement of the final round of each tournament $t$ ). This will later contribute to a spatially autoregressive model that accounts for the strategic interactions.

As our identification strategy will, in part, exploit within-tournament variation in performance and competitor characteristics, the entire (final-round) field of 80 players cannot serve as player $i$ 's relevant competitors. Given the variation in scores we observe however, we do not view this as troubling in the sense of miss-identifying relevant competitors. In defining relevant competitors, we rely on an empirical regularity that we observe in the data that suggests a reasonable rule, in the sense that a player may feasibly surpass (or fall behind) any competitor within his group of relevant competition. Namely, seven strokes is the largest difference in cumulative strokes between any two contiguously ranked players going into the final round of any tournament on the 2004 season of the PGA Tour. As such, at the end of the third round we define $i$ 's relevant competitors as all those with end-of-third-round cumulative performance that is within seven strokes of $i$ 's. ${ }^{16}$

Defining one's relevant competition allows us to test whether the size of one's group of relevant competitors explains one's performance. Additionally, consider the variation introduced into each player's group of competitors by classifying them into categories based upon observable characteristics such as relative standing and ability.

\footnotetext{
${ }^{16}$ We also restrict competitors to have been on the field of play with enough time to respond (i.e., not finishing their round fourth round before a "competitor" even begins play).
} 


\subsubsection{Players' relative standings}

Abstracting away from the two-player tournament, one might reasonably argue that only the effort of the set of $i$ 's relevant competitors need influence $i$ 's marginal probability of winning, where the set of relevant competitors captures all competitors who still have a positive probability of beating $i$ in the tournament conditional on past performance. In that way, one's pattern of play should only be influenced by the play of those within "close" standing. In particular, then, we should anticipate an empirical methodology that is flexible to having certain "close" competitors matter differently than other irrelevant competitors. If a player $j$ is truly irrelevant to the probability of $i$ winning (the corollary in the two-player model being that $F_{e_{i}, e_{j}}=0$ ) then there should be no correlation in their final-round efforts or their observable performance. ${ }^{17}$ Empirically, in each tournament we classify competitors as ahead, tied, or behind based on the rank-order of players at the conclusion of third round play.

\subsubsection{Players' relative abilities}

As an alternative, one might consider the implications of heterogeneity in ability. First, one could allow marginal costs of effort to differ, with the suggestion that players are endowed with different abilities that translate into different effort costs. While it can be shown that each player's best response function is invariant to changes in the opponent's effort costs, a player's own effort (as a best-response, that is) is everywhere lower when effort is more costly. Second, one could model differences in ability, not as differences in marginal costs but, as differences in

\footnotetext{
${ }^{17}$ Of course, if $e_{i}$ and $e_{j}$ are additively separable within the function that determines the probability of winning, implying that $F_{e i, e j}=0$, the reaction function's slope would be zero. However, such a technology seems somewhat implausible as a general rule within the context of explicit tournaments. We will therefore interpret a zero slope as suggestive of no significant strategic interactions between players. There are exceptions to this, however, that seem particularly reasonable given our particular empirical application, each seemingly relating to some dimension of player heterogeneity.
} 
some ceteris paribus probability of winning - essentially, ability differences other than those captured by differences in effort costs. As an extension, then, one might consider the potential for competition among unequal peers to yield private returns to relative performance that go beyond monetary rewards, yielding reward values that are dependent on who has been beaten in the tournament. In particular, it may be reasonable to consider that marginal rewards to outperforming competitors of higher ability (and in that sense, deliver an "upset”) may exceed those that result from out-performing competitors of like or lower ability.

With respect to player ability, we classify relevant competitors based on a comparison of the deciles of players' average scores over all rounds played on the 2003 PGA Tour, with higher deciles corresponding to higher average scores (which should be interpreted as lower average ability). The implication is that $i$ 's opponents with a 2003 scoring average in a lower decile are considered higher ability players (relative to $i$ ). We further classify competitors in the same decile as similar in ability and those in higher deciles as lower ability. Given these discrete classifications, we can further define the intersecting sets as proper sub-groups (i.e., higher ability and ahead, higher ability and behind, etc.).

\subsection{Do players perform differently when facing different types of competitor?}

All together, by introducing asymmetries in effort costs or in rewards that are endogenous to players' relative ex ante abilities, to consider player heterogeneity suggests that an appropriate empirical methodology should be flexible to having certain "higher-ability" or "lower-ability" competitors matter differently than others. In Table 2, we exploit the variation in such characteristics within one's group of relevant competitors and allow player performance to differ with the make-up of this group. In particular, given that each player may face a different number of total competitors within a seven-stroke range, we control for each player's relevant competition 
by including the proportion of $i$ 's relevant competitors falling into each sub-group. Here again, however, the composition of one's relevant group of competitors is not exogenous. Rather, a player's competition at the end of the third round is determined by the cumulative performance of his opponents over the tournament's first three rounds. As with RETURN, we will treat the composition of one's competition as endogenous, using an IV strategy similar to that which we described above. Column (1) of Table 2 delineates sub-groups by ability classifications, with the omitted group capturing the interaction of final-round performance with those competitors with similar ability. Column (2) delineates sub-groups by relative ranking, with the omitted group capturing the interaction of final-round performance with those competitors with equal strokes (as of the end of the third round). Here we learn that, on average, player-performance is better when the field is less similar in ability. The larger is either the proportion of higher or lower-ability competitors the better is performance, on average.

Column (3) allows for the intersection of these groups, controlling for both ability and relative rank. The results in Table 2 provide little evidence that the composition of players' peer groups influences player performance. However, this simple empirical strategy takes no account of the contemporaneous performance of competitors, or changes in group composition during the final round, as the composition of a player's group of relevant competitors is defined at the beginning of the final round. We therefore forego any additional discussion, since such a strategy leaves us unable to appropriately address our primary interest - the interdependency of contemporaneous player behavior in tournaments.

\subsection{Do players perform differently when competitors perform differently?}

Having considered the potential for player performance to vary with the characteristics of the relevant competition, we now reflect on the possible dynamics that produced the data. In 
particular, consider that the fourth round (rather than being a single event) can be divided into 18 sequential holes, each one revealing new information to player $i$ regarding the performance of $i$ 's relevant competition. Thus, even though we as econometricians are unable to observe the variation in performance hole by hole, we suspect that players respond to this information. As we observe only the (more-aggregate) end-of-third- and end-of-fourth-round scores, we must adopt a specification that allows us to sign and measure the correlation between a player's end-of-fourthround score and that of his competitors.

We therefore adopt a spatially autoregressive model of final-round performance that identifies exactly potential patterns underlying the endogenous determination of all fourth-round performances. ${ }^{18}$ Such a model is captured by modifying Eq. [1] as follows:

$$
S=\beta Z+\rho W S+\omega,
$$

where $Z$ captures all right-rand-side variables in [1], $\rho$ is the spatial-lag coefficient to be estimated, and $W$ is the spatial weighting matrix. Given our rule for determining the relevant group of competitors with which one competes, $W$ assigns equal weight to each of $i$ 's tournament$t$ competitors. Thus, $\rho W S$ can be interpreted as the average fourth-round performance of one's relevant competitors.

Of course, given strategic interaction in tournament competition, player scores are jointly determined. Solving [6] for equilibrium player scores yields

$$
S=(I-\rho W)^{-1} \beta Z+(I-\rho W)^{-1} \omega,
$$

in which the random component of the $k^{\text {th }}$ player's score is equal to the inner product of the $k$ th row of the matrix $(I-\rho W)^{-1}$ and the error vector $\omega$. Thus, each player's final-round score is a

\footnotetext{
${ }^{18}$ We do not argue that the fourth-round performance of $i$ and of $i$ 's competition is determined contemporaneously in a literal sense. However, not observing hole-by-hole performance amounts to treating final-round performance as contemporaneously determined.
} 
function of all the $\omega$ 's. Such correlation results in inconsistent OLS estimates of parameters in [6] and therefore requires alternative estimation techniques. Following Anselin (1988), we correct for the endogeneity of player scores with an instrumental variables procedure, in which we regress the $W S$ on $Z$ and $W Z$ and use the fitted values of ${ }_{W} S$ as instruments for WS.

Results in columns (1) and (2) of Table 3 suggest that $\rho$ is positive and significantly different from zero, confirming that a player's realized end-of-fourth-round score does correlate positively with that of his competitors. However, a check for the robustness of the estimated spatial lag suggests that including the weighted average of the absolute performance of a player's competitors may be restrictive. That a player's performance correlates cross-sectionally with the average performance of his relevant competition is consistent with explanations other than the existence of peer effects. ${ }^{19}$ In general, we might expect players of similar ability to be of similar rank at both the end of the third round (where we define them as relevant competitors) and at the end of the fourth round. It is therefore possible that a specification that includes absolute performance, such as in [6], will not permit the separation of the endogenous interdependency between competitors from a general correlation between players of similar ability.

We therefore modify the spatial lag term such that the performance of player $i$ 's group of competitors is measured relative to the competitors' past performance, removing these potential level-effects (i.e., like players performing similarly) and instead capturing whether competitors are gaining or losing ground on player $i$. Defining each player's past performance as the mean strokes per round during the first three rounds of play, we calculate the spatial lag as the average meandifferenced performance over all players in the group. For example, a negative lag in this context

\footnotetext{
${ }^{19}$ Of course, were all players in the fourth round to post scores that were exactly equal to their average over the first three rounds, player performance would correlate (one-to-one) with cross-sectional variation in competitor performance.
} 
will indicate that, on average, the group improved during the fourth round, which, all else equal, implies that relative to his group, a player's cardinal ranking has fallen.

The results from regressions using this group-relative measure of peer performance are shown in columns (3) and (4) of Table 3, where we see again that players respond positively to the performance of relevant competitors, on average. While we have controlled broadly for player ability with the inclusion of players' exemption status and tour affiliations, it would be easily argued that these early specifications do not adequately control for player ability. Thus, we next limit the variation to that specifically around individual players. In so doing we assume that player ability is time-invariant within a single season, after controlling for the trends in performance. Controlling for players' fixed attributes reveals the robustness of the earlier result to a specification where we limit the variation to differences across tournaments for individual players. By including player fixed effects we are exploiting the fact that in each tournament, an individual player will be matched to a different group of relevant competitors, based on relative rank going into the final round. Given that match, each player will have better-performing competitors in some tournaments than in others. Coefficient estimates in Column (4) suggest that individual players perform better when facing better-performing competition. If anything, exploiting only the time-series variation across the multiple tournaments suggests that the strategic interdependency is even stronger. In all subsequent specifications we therefore account for all player-specific heterogeneity.

\subsection{Asymmetric responses to the performance of competitors: Do ability and rank matter?}

Estimation in Table 3 restricts players' responses to competitor performance to be uniform across different types of competitors. It ignores any distinction between better competitors and worse competitors or between leading and lagging competitors. Theory suggests, however, that it 
may be informative to differentiate sub-groups by ability, ordinal rank at the end of the third round, or a combination of both, to which we now turn. In Table 4, we decompose the relevant competitor group into sub-groups based on ability and rank. In considering the ability-breakdown reported in Column (1), note that results suggest that players only respond to the performance of similarly-able competitors and appear unaffected by the performance of either higher-ability or lower-ability competitors. ${ }^{20}$ The point estimate implies that an average one-stroke improvement (i.e., a decline in final-round strokes) in the performance of similarly able competitors elicits an improvement in a player's performance of 0.65 strokes.

In the second column of Table 4 we distinguish sub-groups based on relative rank. In such a specification, competitor-groups that are classified as competing from behind appear to elicit no competitive response in the final round of play. However, point estimates suggest that from within one's group of relevant competitors, there are sizeable responses to the performance of those who are either tied or leading as of the end of round three.

In our most flexible specification, we classify each player's relevant competitors into one of nine sub-groups defined by both their relative rank and relative ability. In Column (3) of Table 4, strategic interactions are significant in six of the nine sub-groups. From these six, with the exception of less-able competitors competing from behind, the correlation in player performance within these sub-groups is positive, suggesting a pattern of behavior that responds to lower scores with lower scores and to higher scores with higher scores.

Consider first players' responses to competitors with whom they are tied. Of those players who are tied, the slope of the best-response function is significantly positive for competitors of similar ability and of higher ability. Notably, the magnitude of the response to similarly-able

\footnotetext{
${ }^{20}$ Or, at least, if they are influenced by individual competitors within the group of relevant competitors, it nets out to no significant dependency overall.
} 
competitors is twice as large as the response to higher-ability competitors, even thought they are both tied. Specifically, a single-stroke improvement in the final round from tied, similarly-able competitors is associated with a player lowering his own score by 0.27 strokes versus only 0.14 strokes for single-stroke improvement from tied, higher-ability competitors. This difference may highlight how competitor characteristics influence the degree to which they are considered "close" peers, the closest peers being those who are similarly able ("close" in a longer-term sense) and also tied going into the final round ("close" in a particular tournament). By extension then, tied, higher-ability competitors are not as close, yet appear sufficiently close to warrant strategic interaction. Thus, players who observe their closest peers improving may be relatively more motivated to lower their scores, simply because they feel they should perform as well as their peers.

Also of note is that, of similarly-able competitors, the average player's response to the performance of those who are tied is larger than the response to the performance of those who are either ahead or behind. This may not be surprising since improvement from tied competitors implies a potential drop in one's rank order and therefore payoff. The same cannot be said, for example, for the improved performance of lagging peers as an individual's payoff is affected only in the case that the improvement results in a tie. Likewise, when leading peers improve, the cardinal separation increases between an individual and his leading peers yet, all else equal, the individual's ordinal rank and payoff remain unchanged.

A slightly different pattern occurs with competitors of similar and lower ability who are ahead in the tournament, though both responses are also positive. Of the observed responses to leading competitors, the competitive response to lower-ability competitors appears considerably larger than the response to similar-ability players. We suspect that asymmetry in this context may 
imply that expectations of competitor performance play a role in effort decisions. For example, the response to lower-ability competitors may be larger than to similarly able competitors if lowerability competitors are expected to have relatively inferior final-round performance, which would tend to decrease their ordinal ranking. By extension, the average player's expected return to effort should be higher in relation to lower-ability, leading competitors. In this sense, the effect of peers being ahead may be offset countered by those peers being of lower ability.

Distinct from those relationships above, players appear to respond quite differently to the performance of competitors who are of lower-ability and are behind. With a negative point estimate, this response is markedly different from the others, implying that the better is the performance of lagging, lower-ability competitors, the worse is a player's own performance. Specifically, a one-stroke improvement in final-round score from this comparator group implies a final-round score that is 0.20 strokes worse (i.e., higher), on average. ${ }^{21}$ This result may imply a level of importance in the psychological effects of being outperformed by players who are behind or less able. Since these competitors are both lower ability (i.e., they tend to perform poorly in a long-term sense) and behind (i.e., are performing poorly in a short-term sense), it is not likely the case that one would be expecting them to improve in the final round. When they do, then, this may amount to psychic costs imposed on others. Perhaps unexpected pressure from these competitors is psychologically disturbing and results in a player posting higher scores.

Given the discrete nature of our scoring data, it is difficult to assign an exact monetary value to the influence of competitors. Rough indicators of this value suggest, however, that the estimated responses above may affect final payoffs quite significantly. For example, while the

\footnotetext{
${ }^{21}$ Recall that a player's improvement is measured against the player's average score over the first three rounds.
} 
median player's potential gain in prize money from improving his final-round score by a single stroke varies considerably across tournaments, the inner-quartile range is $\$ 14,000$ to $\$ 35,000$.

\section{Conclusion}

Existing tournament theory and empirical studies that aim to test such theory largely neglect the issue of player interdependency. Perhaps a main reason for this is that the nonmonotonicity of players' best-response functions in the standard theory results in ambiguous predictions. As the slope of the best-response function is highly sensitive to any additions to the standard model, casting such models into dynamic contexts, for example, often proves intractable. Similarly, even relatively simple modifications of standard tournament models - introducing endowed standings, heterogeneous costs, or heterogeneous ability coupled with endogenous prizes - are sufficient to yield complex relationships between tournament competitors.

Using a spatial econometric approach, we analyze data from tournaments during the 2004 PGA tour and find that players are indeed influenced by their competitors' contemporaneous performance, quite substantially in some cases. In particular, our analysis reveals a tendency for players to respond strongly to the performance of players with whom they are tied going into the final round of competition. This may be expected, as tied competitors are unique insofar as a player's monetary reward must change with either an improvement or decline in the performance of tied competitors, suggesting a larger marginal return with respect to efforts that separate one from a tied competitor. Single-stroke changes in the performance of leading and lagging competitors would not be expected to have the same degree of influence over a player's payoff as such changes need not result in changes to ordinal rankings, on which monetary prizes are determined. Our results are consistent with one experiencing a larger increase in payoff for a single-stroke gain on a tied competitor than for the same gain on a leading competitor. We 
likewise document a general tendency for more-pronounced positive responses where the combination of competitors' rank and ability increase the degree to which they would be considered "close" competitors.

Finally, we find a negative response to performance of lagging, lower-ability players. This finding may result from the influence of other unobservable factors that affect player performance - psychological aspects of tournament competition, perhaps. Future research could be directed toward understanding more fully these psychological factors. In the end, these results suggest that existing models take an over-simplified view of tournaments when players' relative rank and relatively ability are observed but not incorporated. Overall, the significant regularities in the data suggest that social interactions, even those found in a fairly straightforward game, can be rather complex. 


\section{References}

Anselin, L. Spatial Econometrics: Methods and Models, Boston: Kluwer Academic Publishers, 1988.

Aoyagi, M., 2003. Information Feedback in a Dynamic Tournament. Institute of Social and Economic Research Discussion Paper No. 580.

Brock, W. and Durlauf, S., 2001. Discrete Choice with Social Interactions. The Review of Economic Studies, 68, 235-260.

Brueckner, J.K., 1999. Testing for Strategic Interaction Among Local Governments: The Case of Birth Controls. Journal of Urban Economics, 44, 438-467.

Brueckner, J.K., and Saavedra, L.A., 2001. Do Local Governments Engage in Strategic PropertyTax Competition? National Tax Journal, 54, 203-220.

Bull, C., Schotter, A., and Weigelt, K., 1987. Tournaments and Piece Rates: An Experimental Study. Journal of Political Economy, 95, 1-33.

Case, A. and Katz, L., 1991. The Company You Keep: The Effects of Family and Neighborhood on Disadvantaged Families. NBER Working Paper 3705.

Ehrenberg, R., and Bognanno, M., 1990a. The Incentive Effects of Tournaments Revisited: Evidence from the European PGA tour. Industrial and Labor Relations Review, 43, 74s-88s.

Ehrenberg, R., and Bognanno, M., 1990b. Do Tournaments Have Incentive Effects? Journal of Political Economy, 98, 1307-1324.

Glaeser, E., Sacerdote, B., and Sheinkman, J. 1996. Crime and Social Interactions. Quarterly Journal of Economics, 106, 507-548.

Hoxby, C., and Weingarth, G., 2006. Taking Race Out of the Equation: School Reassignment and the Structure of Peer Effects. Harvard University Working Paper.

Lazear, E.P., and Rosen, S., 1981. Rank-Order Tournaments as Optimum Labor Contracts. Journal of Political Economy, 89, 841-64.

Lynch, J.G., 2005. The Effort Effects of Prizes in the Second Half of Tournaments. Journal of Economic Behavior \& Organization, 57, 115-129.

McMillen, D.P., Singell, L.D., Jr., and Waddell, G.R., 2007. Spatial Competition and the Price of College. Economic Inquiry, forthcoming.

Orszag, J.M., 1994. A New Look at Incentive Effects and Golf Tournaments. Economics Letters, 46, 77-88. 
Radner, R., 1985. Repeated Principal-Agent Games with Discounting. Econometrica, 53(5), 1173-1198.

Sacerdote, B., 2001. Peer Effects With Random Assignment: Results for Dartmouth Roommates. Quarterly Journal of Economics, 116.

Steinberg, L., Brown, B., and Dornbusch, S., 1996. Beyond the Classroom. (New York: Simon and Schuster).

Tong, K. and Leung, K., 2002. Tournament as a Motivational Strategy: Extension to Dynamic Situations with Uncertain Duration. Journal of Economic Psychology, 23, 399 - 420.

Tullock, G., 1980. Efficient Rent Seeking. In: Buchanan, J., Tollison, R., Tullock, G. (Eds), Toward a theory of rent seeking society. Texas A\&M University Press, pp. 97-112.

Viladecans-Marsal, E., 2004. Agglomeration Economies and Industrial Location: City-Level Evidence. Journal of Economic Geography, 4(5), 565-82.

Zimmerman, D., 2001. Peer Effects in Higher Education: Evidence from a Natural Experiment, Williams Project on the Economics of Higher Education. The Review of Economics and Statistics, 85(1), 9-23. 
Table 1: The determination of final-round performance.

Dependent Variable: Final-round strokes. In columns (3) and (4), we instrument for player-tournament specific `Return’ using the tournament purse, player's stroke totals from each of first three rounds of the same tournament, and information from the player's 2003 season (i.e., scoring average, fraction of cuts made, scoring average pre-cut, and third-round scoring average). Standard errors are in parentheses.

Player performance $\quad$ Player performance

\begin{tabular}{|c|c|c|c|c|}
\hline \multirow[b]{2}{*}{ Independent Variables } & \multirow[b]{2}{*}{$(1)$} & \multirow[b]{2}{*}{$(2)$} & \multicolumn{2}{|c|}{ (Instrument for RETURN) } \\
\hline & & & (3) & (4) \\
\hline $\begin{array}{l}\text { RETURN (to a one-stroke decrease } \\
\text { in stroke count) }\end{array}$ & $\begin{array}{c}-0.006 * * * \\
(0.002)\end{array}$ & $\begin{array}{l}-0.003^{*} \\
(0.002)\end{array}$ & $\begin{array}{c}-0.016 * * * \\
(0.003)\end{array}$ & $\begin{array}{c}-0.011 * * * \\
(0.003)\end{array}$ \\
\hline Player is exempt & $\begin{array}{c}-0.869 * * \\
(0.185)\end{array}$ & & $\begin{array}{c}-0.751 * * * \\
(0.189)\end{array}$ & \\
\hline Player on Nationwide Tour & $\begin{array}{l}-1.315 \\
(1.541)\end{array}$ & & $\begin{array}{l}-1.430 \\
(1.552)\end{array}$ & \\
\hline Player on Champions Tour & $\begin{array}{l}1.027 \\
(1.023)\end{array}$ & & $\begin{array}{c}0.879 \\
(1.031)\end{array}$ & \\
\hline Course par & $\begin{array}{l}0.478 * * * \\
(0.113)\end{array}$ & $\begin{array}{l}0.465 * * * \\
(0.115)\end{array}$ & $\begin{array}{c}0.467 * * * \\
(0.114)\end{array}$ & $\begin{array}{c}0.459 * * * \\
(0.116)\end{array}$ \\
\hline Course yardage & $\begin{array}{c}0.002 * * * \\
(0.000)\end{array}$ & $\begin{array}{c}0.002 * * * \\
(0.000)\end{array}$ & $\begin{array}{c}0.002 * * * \\
(0.000)\end{array}$ & $\begin{array}{c}0.002^{* * *} \\
(0.000)\end{array}$ \\
\hline Course rating & $\begin{array}{l}-0.056 \\
(0.077)\end{array}$ & $\begin{array}{l}-0.071 \\
(0.078)\end{array}$ & $\begin{array}{l}-0.044 \\
(0.078)\end{array}$ & $\begin{array}{l}-0.062 \\
(0.079)\end{array}$ \\
\hline Week of year & $\begin{array}{c}0.034 \\
(0.028)\end{array}$ & $\begin{array}{c}0.038 \\
(0.029)\end{array}$ & $\begin{array}{c}0.038 \\
(0.028)\end{array}$ & $\begin{array}{c}0.042 \\
(0.029)\end{array}$ \\
\hline$\left(\right.$ Week of year) ${ }^{2}$ & $\begin{array}{c}-0.001 * * \\
(0.001)\end{array}$ & $\begin{array}{c}-0.001 * * \\
(0.001)\end{array}$ & $\begin{array}{c}-0.001 * * * \\
(0.001)\end{array}$ & $\begin{array}{c}-0.001 * * \\
(0.001)\end{array}$ \\
\hline Hot (maximum temp > 90f) & $\begin{array}{c}0.046 \\
(0.325)\end{array}$ & $\begin{array}{l}-0.130 \\
(0.334)\end{array}$ & $\begin{array}{c}0.033 \\
(0.328)\end{array}$ & $\begin{array}{l}-0.137 \\
(0.335)\end{array}$ \\
\hline Cold (maximum temp < 60f) & $\begin{array}{c}-0.685^{* *} \\
(0.338)\end{array}$ & $\begin{array}{c}-0.958^{* * *} \\
(0.346)\end{array}$ & $\begin{array}{l}-0.666^{*} \\
(0.341)\end{array}$ & $\begin{array}{c}-0.928 * * * \\
(0.347)\end{array}$ \\
\hline Maximum wind speed (mph) & $\begin{array}{l}0.089 * * * \\
(0.016)\end{array}$ & $\begin{array}{c}0.081^{* * *} \\
(0.016)\end{array}$ & $\begin{array}{c}0.088 * * * \\
(0.016)\end{array}$ & $\begin{array}{c}0.082 * * * \\
(0.016)\end{array}$ \\
\hline Constant & $\begin{array}{l}26.605 \\
(7.081)\end{array}$ & $\begin{array}{c}29.181 * * * \\
(7.240)\end{array}$ & $\begin{array}{c}24.894 * * * \\
(7.134)\end{array}$ & $\begin{array}{c}29.392 * * * \\
(7.273)\end{array}$ \\
\hline Player Fixed Effects & No & Yes & No & Yes \\
\hline Observations / Unique players & 2221 & $2221 / 227$ & 2221 & $2221 / 227$ \\
\hline
\end{tabular}

${ }^{* * *} \mathrm{p}<0.01,{ }^{* *} \mathrm{p}<0.05,{ }^{*} \mathrm{p}<0.1$. Variables are defined as follows: Return - gain in prize money from improving cardinal rank by one stroke during final round; Exempt - players' exemption status; Nationwide Tour, Champions Tour - player is member of Nationwide Tour/Champions Tour; par, rating - in number of strokes; yardage - in thousands of yards; week of year, (week of year) ${ }^{2}$ - week of the year (and its square) in which the tournament was played; Hot/Cold - indicate whether or not maximum temperature on day of play was greater than 90 and less than 60 degrees Fahrenheit, respectively; Max. Wind speed - maximum sustained wind speed (in mph). 


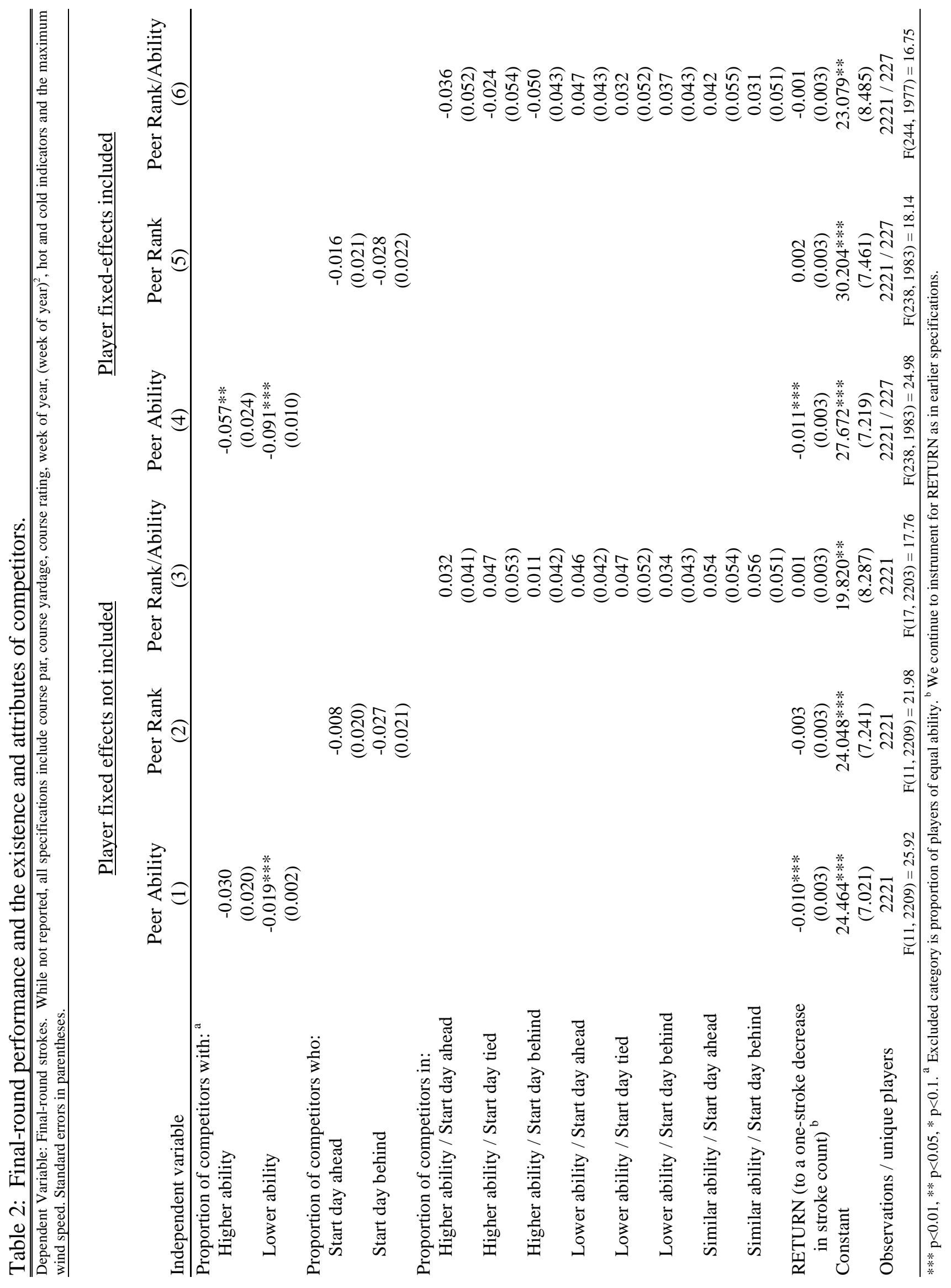




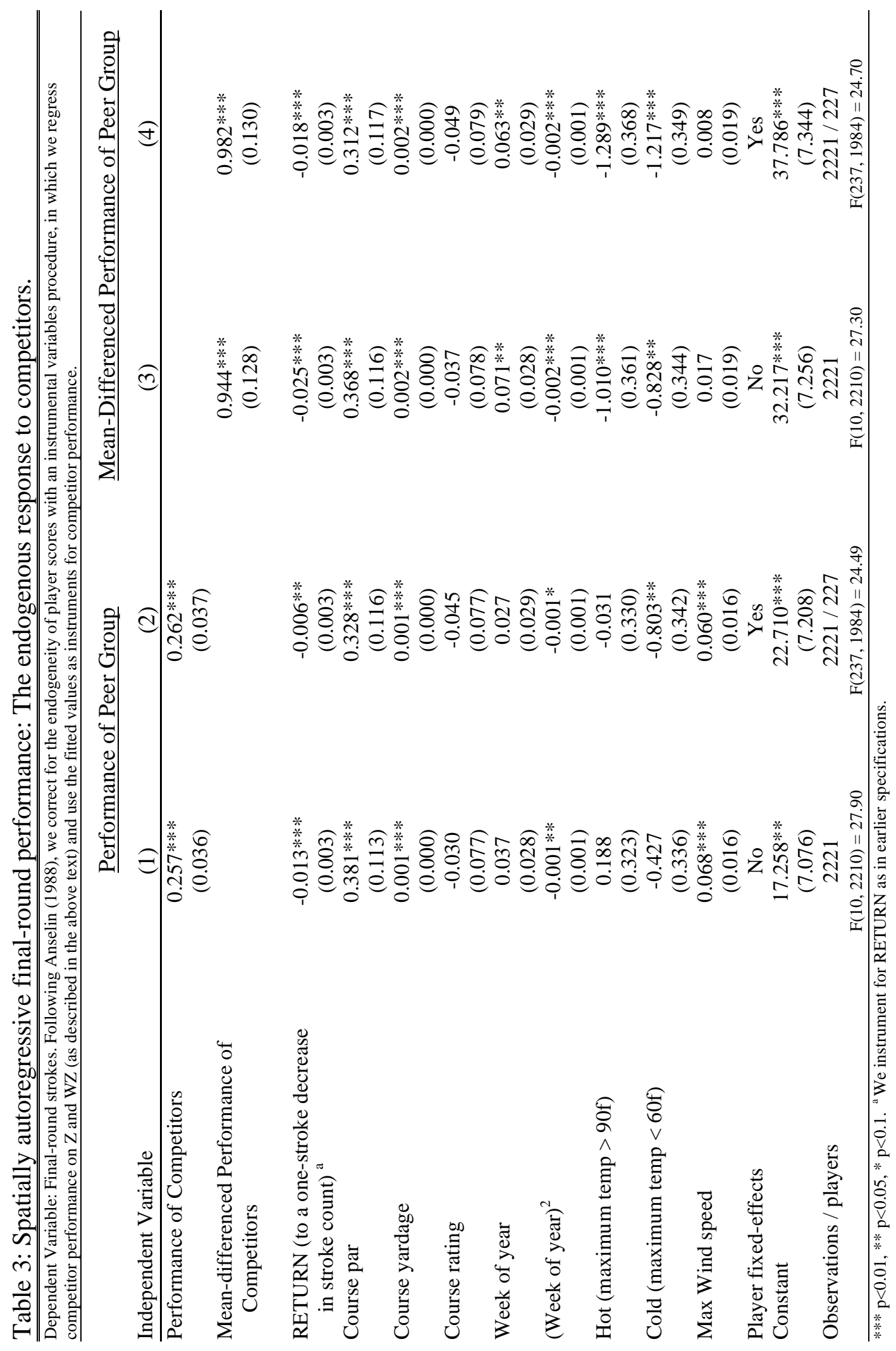


Table 4: Spatially autoregressive performance: Asymmetries in responsiveness to competitor performance.

Dependent Variable: Final-round strokes. Following Anselin (1988), we correct for the endogeneity of player scores with an instrumental variables procedure, in which we regress competitor performance on $\mathrm{Z}$ and WZ (as described in the above text) and use the fitted values as instruments for competitor performance. While estimated coefficients are not reported, all specifications include course par, course yardage, course rating, week of year, (week of year) ${ }^{2}$, hot and cold indicators and maximum wind speed.

\begin{tabular}{|c|c|c|c|}
\hline Independent Variable & $(1)$ & $(2)$ & $(3)$ \\
\hline \multicolumn{4}{|l|}{ Performance of relevant competition categorized as: } \\
\hline \multirow[t]{2}{*}{ Lower Ability } & 0.118 & & \\
\hline & $(0.130)$ & & \\
\hline \multirow[t]{2}{*}{ Similar Ability } & $0.645 * * *$ & & \\
\hline & $(0.100)$ & & \\
\hline \multirow[t]{2}{*}{ Higher Ability } & 0.146 & & \\
\hline & $(0.104)$ & & \\
\hline \multirow[t]{2}{*}{ Behind } & & -0.169 & \\
\hline & & $(0.108)$ & \\
\hline \multirow[t]{2}{*}{ Tied } & & $0.292 * *$ & \\
\hline & & $(0.099)$ & \\
\hline \multirow[t]{2}{*}{ Ahead } & & $0.298 * * *$ & \\
\hline & & $(0.095)$ & \\
\hline \multirow[t]{2}{*}{ Lower Ability / Behind } & & & $-0.200 * *$ \\
\hline & & & $(0.084)$ \\
\hline \multirow[t]{2}{*}{ Similar Ability / Behind } & & & $0.116^{*}$ \\
\hline & & & $(0.064)$ \\
\hline \multirow[t]{2}{*}{ Higher Ability / Behind } & & & 0.032 \\
\hline & & & $(0.074)$ \\
\hline \multirow[t]{2}{*}{ Lower Ability / Tied } & & & -0.033 \\
\hline & & & $(0.072)$ \\
\hline \multirow[t]{2}{*}{ Similar Ability / Tied } & & & $0.267 * * *$ \\
\hline & & & $(0.077)$ \\
\hline \multirow[t]{2}{*}{ Higher Ability / Tied } & & & $0.137 * *$ \\
\hline & & & $(0.061)$ \\
\hline \multirow[t]{2}{*}{ Lower Ability / Ahead } & & & $0.293 * * *$ \\
\hline & & & $(0.079)$ \\
\hline \multirow[t]{2}{*}{ Similar Ability / Ahead } & & & $0.189 * * *$ \\
\hline & & & $(0.056)$ \\
\hline \multirow[t]{2}{*}{ Higher Ability / Ahead } & & & -0.059 \\
\hline & & & $(0.059)$ \\
\hline \multirow[t]{2}{*}{ RETURN (to a one-stroke decrease in stroke count) ${ }^{a}$} & $-0.009 * * *$ & $-0.009 * * *$ & $-0.005^{*}$ \\
\hline & $(0.003)$ & $(0.003)$ & $(0.003)$ \\
\hline Player fixed-effects & Yes & Yes & Yes \\
\hline \multirow[t]{2}{*}{ Constant } & $37.993 * * *$ & $27.869 * * *$ & $31.213^{* * *}$ \\
\hline & $(7.557)$ & $(7.504)$ & $(7.624)$ \\
\hline \multirow[t]{2}{*}{ Observations } & $2221 / 227$ & $2221 / 227$ & $2221 / 227$ \\
\hline & $F(239,1982)=22.00$ & $\mathrm{~F}(239,1982)=18.36$ & $F(245,1976)=14.68$ \\
\hline
\end{tabular}

Standard errors in parentheses. ${ }^{* * *} \mathrm{p}<0.01,{ }^{* *} \mathrm{p}<0.05,{ }^{*} \mathrm{p}<0.1{ }^{\mathrm{a}}{ }^{\mathrm{a}}$ We instrument for RETURN as in earlier specifications. 